Research Article

\title{
Certain Properties of Generalized $M$-Series under Generalized Fractional Integral Operators
}

\author{
D. L. Suthar $(\mathbb{D}$, Fasil Gidaf $(\mathbb{D}$, and Mitku Andualem \\ Department of Mathematics, Wollo University, P.O. Box: 1145, Dessie, Ethiopia \\ Correspondence should be addressed to D. L. Suthar; dlsuthar@gmail.com
}

Received 2 March 2021; Revised 13 June 2021; Accepted 25 June 2021; Published 6 July 2021

Academic Editor: Ahmet Ocak Akdemir

Copyright (C) 2021 D. L. Suthar et al. This is an open access article distributed under the Creative Commons Attribution License, which permits unrestricted use, distribution, and reproduction in any medium, provided the original work is properly cited.

The aim of this study is to introduce new (presumed) generalized fractional integral operators involving $I$-function as a kernel. In addition, two theorems have been developed under these operators that provide an image formula for this generalized $M$-series and also to study the different properties of the generalized $M$-series. The corresponding assertions in terms of Euler and Laplace transform methods are presented. Due to the general nature of the $I$-function and the generalized $M$-series, a number of results involving special functions can be achieved only by making appropriate values for the parameters.

\section{Introduction}

Recently, in a short note, Sharma and Jain [1] introduced and studied a new special function called as generalized $M$-series, which is a particular case of the Wright generalized hypergeometric function ${ }_{p} \psi_{q}($.$) ([2], p. 56, equation$ (1.11.14)) and Fox's $H$-function [3-5]. The generalized $M$-series is important because its basic cases are followed by the Mittag-Leffler function and hypergeometric function, and all these functions have actually discovered key implementations in solving problems in applied sciences, chemistry, physics, and biology. A number of researchers [6-10] have also investigated the structure, implementations, and various directions of extensions of the fractional integration and differentiation in detail. The series is defined for $z, \varphi, \varsigma \in \mathbb{C}, \mathfrak{R}(\varphi)>0$, and $\alpha_{i}, \beta_{j} \in \mathbb{R}(-\infty, \infty), \quad\left(\alpha_{i}: i=\right.$ $\left.1,2, \ldots, p ; \beta_{j} \neq 0: j=1,2, \ldots, q\right)$ as

$$
{ }_{p}^{\varphi, \varsigma} M_{q}(z)={ }_{p}^{\varphi, \zeta}\left(\alpha_{q}, \ldots, \alpha_{p} ; \beta_{1}, \ldots, \beta_{q} ; z\right)=\sum_{k=0}^{\infty} \frac{\left(\alpha_{1}\right)_{k} \ldots\left(\alpha_{p}\right)_{k}}{\left(\beta_{1}\right)_{k} \ldots\left(\beta_{q}\right)_{k}} \frac{z^{k}}{\Gamma(\varphi k+\varsigma)}
$$

where $\left(\alpha_{j}\right)_{k},\left(\beta_{j}\right)_{k}$ are showing the results for Pochhammer symbols. The series (1) is defined when none of the parameters $\beta_{j} s,(j=1, \ldots, q)$ is a negative integer or zero; if any numerator parameter $\alpha_{j}$ is a negative integer or zero, then the series terminates to a polynomial in $z$. The series in (1) is convergent for all $z$ if $p \leq q$, it is convergent for $|z|<\vartheta=$ $\varphi^{\varphi}$ if , and it is divergent if $p>q+1$. When $p=q+1$ and $|z|<\vartheta$, the series can converge on conditions depending on the parameters ([2], for the general theory of the Wright function). The summation of the convergent series is denoted by the symbol ${ }_{p}^{\varphi, \varsigma} M_{q}($.$) .$

Some essential special cases of the generalized $M$-series are mentioned in the following:

(1) For $\varphi=\varsigma=1$, the generalized $M$-series is the generalized hypergeometric function $[11,12]$. 


$$
{ }_{p} F_{q}\left[\begin{array}{l}
\alpha_{1}, \ldots, \alpha_{p} \\
\beta_{1}, \ldots, \beta_{q}
\end{array} z\right]={ }_{p}^{1,1} \stackrel{M}{q}_{q}\left(\alpha_{1}, \ldots, \alpha_{p} ; \beta_{1}, \ldots, \beta_{q} ; z\right)=\sum_{k=0}^{\infty} \frac{\left(\alpha_{1}\right)_{k} \ldots\left(\alpha_{p}\right)_{k}}{\left(\beta_{1}\right)_{k} \ldots\left(\beta_{q}\right)_{k}} \frac{z^{k}}{k !}
$$

(2) When $p=q=0$ and $\varsigma=1$, we have

$$
E_{\varphi}(z)={ }_{0}^{\varphi, 1} M_{0}(-;-; z)=\sum_{k=0}^{\infty} \frac{z^{k}}{\Gamma(\varphi k+1)},(\varphi>0)
$$

Where the symbol $E_{\varphi}(z)$ denotes the Mittag-Leffler function [13].

(3) Again, for $p=q=0$, we have

$$
E_{\varphi, \varsigma}(z)={ }_{0} \stackrel{\varphi, \varsigma}{M}_{0}(-;-; z)=\sum_{k=0}^{\infty} \frac{z^{k}}{\Gamma(\varphi k+\varsigma)},(\varphi>0, \varsigma>0),
$$

Where the symbol $E_{\varphi, \varsigma}(z)$ denotes the two-index Mittag-Leffler function introduced by Wiman [14].

(4) Furthermore, if we put $p=q=1, \alpha_{1}=\sigma \in \mathbb{C}, \beta_{1}=1$, the generalized $M$-series reduces to the generalized Mittag-Leffler function [12, 15] as follows:

$$
\begin{aligned}
E_{\varphi, \zeta}^{\sigma}(z) & ={ }_{1}^{\varphi, \zeta} M_{1}(\sigma ; 1 ; z)=\sum_{k=0}^{\infty} \frac{(\sigma)_{k}}{(1)_{k}} \frac{z^{k}}{\Gamma(\varphi k+\varsigma)} \\
& =\sum_{k=0}^{\infty} \frac{(\sigma)_{k}}{\Gamma(\varphi k+\varsigma)} \frac{z^{k}}{k !} .
\end{aligned}
$$

In the present study, our aim is to study some fundamental properties of generalized $M$-series defined by (1), for which, we consider the two generalized fractional integral operators involving the $I$-function as kernel, which is described in the next section.

\section{Generalized Fractional Integral Operators}

In this section, we are introducing new (presumed) generalized fractional integral operators involving $I$-function as kernel, which are the extensions of Saxena and Kumbhat operators $[16,17]$ :

$$
S_{0, x ; r}^{\mu, 9}[f(x)]=r x^{-\mu-r 9-1} \int_{0}^{x} t^{\mu}\left(x^{r}-t^{r}\right)^{9} \times I_{p_{i}, q_{i}: r}^{m, n}\left[\begin{array}{c}
\left(a_{j}, A_{j}\right)_{1, n} ;\left(a_{j i}, A_{j i}\right)_{n+1, p_{i}} \\
\left(b_{j}, B_{j}\right)_{1, m} ;\left(b_{j i}, B_{j i}\right)_{m+1, q_{i}}
\end{array}\right] f(t) \mathrm{d} t
$$

and

$$
S_{x, \infty ; r}^{\varepsilon, 9}[f(x)]=r x^{\varepsilon} \int_{x}^{\infty} t^{-\varepsilon-r 9-1}\left(t^{r}-x^{r}\right)^{9} \times I_{p_{i}, q_{i}: r}^{m, n}\left[\begin{array}{c}
\left(a_{j}, A_{j}\right)_{1, n} ;\left(a_{j i}, A_{j i}\right)_{n+1, p_{i}} \\
\left(b_{j}, B_{j}\right)_{1, m} ;\left(b_{j i}, B_{j i}\right)_{m+1, q_{i}}
\end{array}\right] f(t) \mathrm{d} t,
$$

where

$$
U=\left(\frac{t^{r}}{x^{r}}\right)^{\tau}\left(1-\frac{t^{r}}{x^{r}}\right)^{v} \text { and } V=\left(\frac{x^{r}}{t^{r}}\right)^{\tau}\left(1-\frac{x^{r}}{t^{r}}\right)^{v} ; \quad \tau, \nu>0 .
$$

The sufficient conditions of these operators are

$$
\begin{aligned}
\text { (i) } & 1 \leq p, q<\infty, p^{-1}+q^{-1}=1 ; \\
\text { (ii) } & \Re\left(\mu+r \tau\left(b_{j} / B_{j}\right)\right)>-q^{-1} ; \Re\left(\vartheta+r \tau\left(b_{j} / B_{j}\right)\right)> \\
& \quad-q^{-1} ; \\
& \Re\left(\varepsilon+\vartheta+r \tau\left(\frac{b_{j}}{B_{j}}\right)\right)>-p^{-1} ; \quad j=1,2, \ldots, m .
\end{aligned}
$$

(iii) $f(x) \in L_{p}(0, \infty)$

(iv) $|\arg \lambda| \leq \pi \Theta / 2, \Theta>0$

$$
\Theta=\sum_{j=1}^{m}\left(A_{i}\right)+\sum_{j=1}^{n}\left(B_{i}\right)-\max _{1 \leq i \leq r}\left[\sum_{j=n+1}^{p_{i}}\left(A_{j i}\right)+\sum_{j=m+1}^{q_{i}}\left(B_{j i}\right)\right] .
$$

where the $I$-function, which is more general than Fox's $H$-function, is defined by Saxena [18], by means of the following Mellin-Barnes type contour integral:

$$
I[z]=I_{p_{i}, q_{i} ; r}^{m, r}\left[\begin{array}{c}
\left(a_{j}, A_{j}\right)_{1, n} ;\left(a_{j i}, A_{j i}\right)_{n+1, p_{i}} \\
\left(b_{j}, B_{j}\right)_{1, m} ;\left(b_{j i}, B_{j i}\right)_{m+1, q_{i}}
\end{array}\right]=\frac{1}{2 \pi \omega} \int_{L} \varphi(\zeta) z^{\zeta} \mathrm{d} \zeta,
$$

where $\omega=\sqrt{-1}$ and 


$$
\varphi(\zeta)=\prod_{j=1}^{m} \Gamma\left(b_{j}-B_{j} \zeta\right) \frac{\prod_{j=1}^{n} \Gamma\left(1-a_{j}+A_{j} \zeta\right)}{\sum_{j=1}^{r}\left\{\prod_{j=m+1}^{q_{i}} \Gamma\left(1-b_{j i}+B_{j i} \zeta\right) \prod_{j=n+1}^{p_{i}}\left(a_{j i}-A_{j i} \zeta\right)\right\}},
$$

$p_{i}, q_{i}(i=1, \ldots, r), m, n$ are the integers satisfying $0 \leq n \leq p_{i}, 0 \leq m \leq q_{i} ; A_{j}, B_{j}, A_{j i}, B_{j i}$ are the real and positive numbers, and $a_{j}, b_{j}, a_{j i}, b_{j i}$ are the complex numbers. $L$ is a suitable contour of the Mellin-Barnes type running from $\gamma-$ $i \varphi$ to $\gamma+i \varphi$ ( $\gamma$ is real) in the complex $\zeta$-plane. Details regarding existence conditions and various parametric restrictions of $I$-function are provided by Saxena [18].

For $r=1$, (11) reduces to Fox' $H$-function:

$$
I_{p_{i}, q_{i} ;}^{m, n}\left[\begin{array}{c}
\left(a_{j}, A_{j}\right)_{1, n} ;\left(a_{j i}, A_{j i}\right)_{n+1, p_{i}} \\
\left(b_{j}, B_{j}\right)_{1, m} ;\left(b_{j i}, B_{j i}\right)_{m+1, q_{i}}
\end{array}\right]=H_{p_{i}, q_{i}}^{m, n}\left[\begin{array}{c}
\left(a_{j}, A_{j}\right)_{1, n} ;\left(a_{j}, A_{j}\right)_{n+1, p} \\
\left(b_{j}, B_{j}\right)_{1, m} ;\left(b_{j}, B_{j}\right)_{m+1, q}
\end{array}\right] .
$$

\section{Images of Generalized $M$-Series under the Generalized Fractional Integral Operators}

In this section, we established the image formula for the generalized $M$-series (1) under the generalized fractional integral operators (6) and (7) in terms of the $I$-function as the kernel. The results are given in Theorems 1 and 2 .

Theorem 1. Let $a>0, x>0 ; \varphi, \zeta, \eta, \xi \in \mathbb{C}, \mathfrak{R}(\varphi)>0, \mathfrak{R}$ $(\xi)>0, \mathfrak{R}(\eta)>0, \mathfrak{R}(\varsigma)>0,1 \leq p \leq 2$, then

$$
\begin{aligned}
& S_{0, x ; r}^{\mu, 9}\left(t_{p}^{\eta-1}{ }_{p}^{\varphi, \varsigma}{ }_{q}\left(a t^{\xi}\right)\right)(x)= x^{\eta-1} \sum_{k=0}^{\infty} \frac{\left(\alpha_{1}\right)_{k} \ldots\left(\alpha_{p}\right)_{k}}{\left(\beta_{1}\right)_{k} \ldots\left(\beta_{q}\right)_{k}} \frac{\left(a x^{\xi}\right)^{k}}{\Gamma(\varphi k+\varsigma)} \\
& \times I_{p+2, q+1 ; r}^{m, n+2}\left[\begin{array}{l}
\left.\lambda \mid\left(a_{j}, A_{j}\right)_{1, n} ;\left(a_{j i}, A_{j i}\right)_{n+1, p_{i}},\left(1-\frac{(\mu+\eta+\xi k)}{r}, \tau\right),(-\vartheta, v)\right] \\
\left(-\vartheta-\frac{(\mu+\eta+\xi k)}{r}, \tau+v\right),\left(b_{j}, \varsigma_{j}\right)_{1, m} ;\left(b_{j i}, \varsigma_{j i}\right)_{m+1, q_{i}}
\end{array}\right],
\end{aligned}
$$

provided the conditions, stated with operator (6), are satisfied.

Proof. We assume $\Omega_{1}$ be the on the left-hand side of (14); using the definition of generalized $M$-series (1) and the generalized fractional integral operator (6) on the left-hand side of (14), we have

$$
\Omega_{1}=r x^{-\mu-r \vartheta-1} \int_{0}^{x} t^{\mu+\eta-1}\left(x^{r}-t^{r}\right)^{\vartheta}\left\{\frac{1}{2 \pi \omega} \int_{l} \varphi(\zeta)(\lambda U)^{\zeta} \mathrm{d} \zeta\right\} \times \sum_{k=0}^{\infty} \frac{\left(\alpha_{1}\right)_{k} \cdots\left(\alpha_{p}\right)_{k}}{\left(\beta_{1}\right)_{k} \ldots\left(\beta_{q}\right)_{k}} \frac{(a)^{\xi}}{\Gamma(\varphi k+\varsigma)} \mathrm{d} t
$$

Now, by changing the order of the integration which is valid under the given with theorem, we get

$$
\Omega_{1}=r x^{-\mu-r 9-1} \sum_{k=0}^{\infty} \frac{\left(\alpha_{1}\right)_{k} \ldots\left(\alpha_{p}\right)_{k}}{\left(\beta_{1}\right)_{k} \ldots\left(\beta_{q}\right)_{k}} \frac{a^{k}}{\Gamma(\varphi k+\varsigma)} \times \frac{1}{2 \pi \omega} \int_{L} \varphi(\zeta) \lambda^{\zeta} x^{r 9-r \tau \zeta}\left\{\int_{0}^{x} t^{\mu+\eta+\xi k+r \tau \zeta-1}\left(1-\frac{t^{r}}{x^{r}}\right)^{9+v \zeta} \mathrm{d} t\right\} \mathrm{d} \zeta
$$

Let the substitution $t^{r} / x^{r}=w$ and then $t=x w^{1 / r}$ in (16), we get 


$$
\Omega_{1}=x^{\eta-1} \sum_{k=0}^{\infty} \frac{\left(\alpha_{1}\right)_{k} \ldots\left(\alpha_{p}\right)_{k}}{\left(\beta_{1}\right)_{k} \cdots\left(\beta_{q}\right)_{k}} \frac{\left(a x^{\xi}\right)^{k}}{\Gamma(\varphi k+\varsigma)} \times \frac{1}{2 \pi \omega} \int_{L} \varphi(\zeta) \lambda^{\zeta}\left\{\int_{0}^{1} w^{((\mu+\eta+\xi k) / r+\tau \zeta)-1}(1-w)^{9+v \zeta} \mathrm{d} w\right\} \mathrm{d} \zeta
$$

Using the definition of the well-known beta function in the inner integral, we have

$$
\Omega_{1}=x^{\eta-1} \sum_{k=0}^{\infty} \frac{\left(\alpha_{1}\right)_{k} \ldots\left(\alpha_{p}\right)_{k}}{\left(\beta_{1}\right)_{k} \ldots\left(\beta_{q}\right)_{k}} \frac{\left(a x^{\xi}\right)^{k}}{\Gamma(k+\varsigma)} \frac{1}{2 \pi \omega} \int_{L} \varphi(\zeta) \lambda^{\zeta} \frac{\Gamma(\mu+\eta+\xi k / r+\tau \zeta) \Gamma(1+\vartheta+v \zeta)}{\Gamma(1+\vartheta+\mu+\eta+\xi k / r+(\tau+v) \zeta)} \mathrm{d} \zeta .
$$

Interpreting the right-hand side of (18), in view of the definition (11), we arrive at the result (14).
Theorem 2. Let $a>0, x>0 ; \varphi, \varsigma, \eta, \xi \in \mathbb{C}, \mathfrak{R}(\varphi)>0, \mathfrak{R}$ $(\xi)>0, \mathfrak{R}(\eta)>0, \mathfrak{R}(\varsigma)>0,1 \leq p \leq 2$, then

$$
\begin{aligned}
& S_{x, \infty ; r}^{\mathcal{\varepsilon}, 9}\left(t_{p}^{-\eta}{ }_{p}^{\varphi, \varsigma} M_{q}\left(\frac{a}{t^{\xi}}\right)\right)(x)= x^{-\eta} \sum_{k=0}^{\infty} \frac{\left(\alpha_{1}\right)_{k} \ldots\left(\alpha_{p}\right)_{k}}{\left(\beta_{1}\right)_{k} \ldots\left(\beta_{q}\right)_{k}} \frac{\left(a / x^{\xi}\right)^{k}}{\Gamma(\varphi k+\varsigma)} \\
& \times I_{p+2, q+1 ; r}^{m, n+2}\left[\begin{array}{l}
\lambda \mid\left(a_{j}, A_{j}\right)_{1, n} ;\left(a_{j i}, A_{j i}\right)_{n+1, p_{i}},\left(1-\frac{(\varepsilon+\eta+\xi k)}{r}, \tau\right),(-\vartheta, v) \\
\left(-\vartheta-\frac{(\varepsilon+\eta+\xi k)}{r}, \tau+v\right),\left(b_{j}, \varsigma_{j}\right)_{1, m} ;\left(b_{j i}, \varsigma_{j i}\right)_{m+1, q_{i}}
\end{array}\right],
\end{aligned}
$$

provided the conditions, stated with operator (7), are satisfied.

Proof. On the left-hand side of (19), let $\Omega_{2}$, using (1) and (7) on the left-hand side of (19), we have

$$
\Omega_{2}=r x^{\varepsilon} \int_{x}^{\infty} t^{-\varepsilon-\eta-r \vartheta-1}\left(t^{r}-x^{r}\right)^{\vartheta}\left\{\frac{1}{2 \pi \omega} \int_{L} \varphi(\zeta)(\lambda V)^{\zeta} \mathrm{d} \zeta\right\} \times \sum_{k=0}^{\infty} \frac{\left(\alpha_{1}\right)_{k} \cdots\left(\alpha_{p}\right)_{k}}{\left(\beta_{1}\right)_{k} \ldots\left(\beta_{q}\right)_{k}} \frac{\left(a / t^{\xi}\right)^{k}}{\Gamma(\varphi k+\zeta)} \mathrm{d} t
$$

Now, by changing the order of the integration which is valid under the given stated theorem, we get

$$
\Omega_{2}=r x^{\varepsilon} \sum_{k=0}^{\infty} \frac{\left(\alpha_{1}\right)_{k} \cdots\left(\alpha_{p}\right)_{k}}{\left(\beta_{1}\right)_{k} \cdots\left(\beta_{q}\right)_{k}} \frac{a^{k}}{\Gamma(\varphi k+\varsigma)} \times \frac{1}{2 \pi \omega} \int_{L} \varphi(\zeta) \lambda^{\zeta} x^{r \tau \zeta}\left\{\int_{x}^{\infty} t^{-\varepsilon-\eta-\xi k-r \tau \zeta-1}\left(1-\frac{x^{r}}{t^{r}}\right)^{9+v \zeta} \mathrm{d} t\right\} \mathrm{d} \zeta
$$

Let the replacement $x^{r} / t^{r}=w$ and then $t=x / w^{1 / r}$ in (21), we get

$$
\Omega_{2}=x^{-\eta-1} \sum_{k=0}^{\infty} \frac{\left(\alpha_{1}\right)_{k} \ldots\left(\alpha_{p}\right)_{k}}{\left(\beta_{1}\right)_{k} \ldots\left(\beta_{q}\right)_{k}} \frac{a^{k} x^{-\xi k}}{\Gamma(\varphi k+\zeta)} \times \frac{1}{2 \pi \omega} \int_{L} \varphi(\zeta) \lambda^{\zeta}\left\{\int_{0}^{1} w^{(\varepsilon+\eta+\xi k / r)+\tau \zeta-1}(1-w)^{9+v \zeta} \mathrm{d} w\right\} \mathrm{d} \zeta .
$$

By beta function, we have 


$$
\Omega_{2}=x^{-\eta+r \varphi} \sum_{k=0}^{\infty} \frac{\left(\alpha_{1}\right)_{k} \ldots\left(\alpha_{p}\right)_{k}}{\left(\beta_{1}\right)_{k} \ldots\left(\beta_{q}\right)_{k}} \frac{\left(a x^{-\xi}\right)^{k}}{\Gamma(\varphi k+\zeta)} \frac{1}{2 \pi \omega} \int_{L} \varphi(\zeta) \lambda^{\zeta} \times \frac{\Gamma((\varepsilon+\eta+\xi k / r)+\tau \zeta) \Gamma(1+\vartheta+v \zeta)}{\Gamma((\varepsilon+\eta+\xi k / r)+1+\vartheta+(\tau+v) \zeta)} \mathrm{d} \zeta .
$$

Interpreting the right-hand side of (23), in view of the definition (11), we arrive at the result (19).

\section{Special Cases}

(1) If we put $\varphi=\varsigma=1$ in Theorems 1 and 2 , we obtain the following interesting results on the right, and it is known as the generalized hypergeometric function.
Corollary 1. For $\varphi=\varsigma=1$, equation (14) reduces in the following form:

$$
\begin{aligned}
& S_{0, x ; r}^{\mu, \vartheta}\left(t^{\eta-1}{ }_{p}^{1,1} M_{q}\left(\alpha_{1}, \ldots, \alpha_{p} ; \beta_{1}, \ldots, \beta_{q} ; a t^{\xi}\right)\right)(x)=\left.x^{\eta-1}{ }_{p} F_{q}\left[\begin{array}{l}
\alpha_{1}, \ldots, \alpha_{p} \\
\beta_{1}, \ldots, \beta_{q}
\end{array}\right] a x^{\xi}\right] \\
& \times I_{p+2, q+1 ; r}^{m, n+2}\left[\begin{array}{l}
\lambda l\left(a_{j}, A_{j}\right)_{1, n} ;\left(a_{j i}, A_{j i}\right)_{n+1, p_{i}},\left(1-\frac{(\mu+\eta+\xi k)}{r}, \tau\right),(-\vartheta, v) \\
\left(-\vartheta-\frac{(\mu+\eta+\xi k)}{r}, \tau+v\right),\left(b_{j}, \varsigma_{j}\right)_{1, m} ;\left(b_{j i}, \varsigma_{j i}\right)_{m+1, q_{i}}
\end{array}\right] .
\end{aligned}
$$

Corollary 2. For $\varphi=\varsigma=1$, equation (19) reduces in the following form:

$$
\begin{aligned}
& S_{x, \infty ; r}^{\varepsilon, 9}\left(t^{-\eta}{ }_{p}^{1,1} M_{q}\left(\alpha_{1}, \ldots, \alpha_{p} ; \beta_{1}, \ldots, \beta_{q} ; \frac{a}{t^{\xi}}\right)\right)(x)= x_{p}^{-\eta} F_{q}\left[\begin{array}{l}
\alpha_{1}, \ldots, \alpha_{p} ; \frac{a}{\beta_{1}, \ldots, \beta_{q}} x^{\xi}
\end{array}\right] \\
& \times I_{p+2, q+1 ; r}^{m, n+2}\left[\begin{array}{l}
\lambda \mid\left(a_{j}, A_{j}\right)_{1, n} ;\left(a_{j i}, A_{j i}\right)_{n+1, p_{i}}\left(1-\frac{(\varepsilon+\eta+\xi k)}{r}, \tau\right),(-\vartheta, v) \\
\left(-9-\frac{(\varepsilon+\eta+\xi k)}{r}, \tau+v\right),\left(b_{j}, \varsigma_{j}\right)_{1, m} ;\left(b_{j i}, \varsigma_{j i}\right)_{m+1, q_{i}}
\end{array}\right] .
\end{aligned}
$$


(2) If we put $p=q=0$ in Theorems 1 and 2, we obtain the following interesting results on the right, and it is known as the two-index Mittag-Leffler function.
Corollary 3. For $p=q=0$, equation (16) reduces in the following form:

$$
\begin{aligned}
& S_{0, x ; r}^{\mu, \vartheta}\left(t^{\eta-1}{ }_{0}^{\varphi, \varsigma} M_{0}\left(-;-; a t^{\xi}\right)\right)(x)=x^{\eta-1} E_{\varphi, \varsigma}\left(a x^{\xi}\right) \\
& \times I_{p+2, q+1 ; r}^{m, n+2}\left[\begin{array}{c}
\lambda \mid\left(a_{j}, A_{j}\right)_{1, n} ;\left(a_{j i}, A_{j i}\right)_{n+1, p_{i}},\left(1-\frac{(\mu+\eta+\xi k)}{r}, \tau\right),(-\vartheta, v) \\
\left(-\vartheta-\frac{(\mu+\eta+\xi k)}{r}, \tau+v\right),\left(b_{j}, \varsigma_{j}\right)_{1, m} ;\left(b_{j i}, \varsigma_{j i}\right)_{m+1, q_{i}}
\end{array}\right] .
\end{aligned}
$$

Corollary 4. For $p=q=0$, equation (19) reduces in the following form:

$$
\begin{aligned}
& S_{x, \infty ; r}^{\mathcal{\varepsilon}, \vartheta}\left(t^{-\eta}{ }_{0}^{\varphi, \varsigma} M_{0}\left(-;-; \frac{a}{t^{\xi}}\right)\right)(x)=x^{-\eta} E_{\varphi, \varsigma}\left(\frac{a}{x^{\xi}}\right) \\
& \times I_{p+2, q+1 ; r}^{m, n+2}\left[\begin{array}{c}
\lambda \mid\left(a_{j}, A_{j}\right)_{1, n} ;\left(a_{j i}, A_{j i}\right)_{n+1, p_{i}},\left(1-\frac{(\varepsilon+\eta+\xi k)}{r}, \tau\right),(-\vartheta, v) \\
\left(-\vartheta-\frac{(\varepsilon+\eta+\xi k)}{r}, \tau+v\right),\left(b_{j}, \varsigma_{j}\right)_{1, m} ;\left(b_{j i}, \varsigma_{j i}\right)_{m+1, q_{i}}
\end{array}\right] .
\end{aligned}
$$

(3) If we put $p=q=1, \alpha_{1}=\sigma \in \mathbb{C}, \beta_{1}=1$ in Theorems 1 and 2 , we obtain the following interesting results on the right, and it is known as the generalized MittagLeffler function.
Corollary 5. For $p=q=1, a_{1}=\xi \in \mathbb{C}, b_{1}=1$, equation (14) reduces in the following form:

$$
\begin{aligned}
& S_{0, x ; r}^{\mu, \vartheta}\left(t^{\eta-1}{ }_{1} \stackrel{\varphi, \zeta}{M}_{1}\left(\sigma ; 1 ; a t^{\xi}\right)\right)(x)=x^{\eta-1} E_{\varphi, \zeta}^{\sigma}\left(a x^{\xi}\right) \\
& \times I_{p+2, q+1 ; r}^{m, n+2}\left[\begin{array}{c}
\lambda \mid\left(a_{j}, A_{j}\right)_{1, n} ;\left(a_{j i}, A_{j i}\right)_{n+1, p_{i}},\left(1-\frac{(\mu+\eta+\xi k)}{r}, \tau\right),(-\vartheta, v) \\
\left(-\vartheta-\frac{(\mu+\eta+\xi k)}{r}, \tau+v\right),\left(b_{j}, \varsigma_{j}\right)_{1, m} ;\left(b_{j i}, \varsigma_{j i}\right)_{m+1, q_{i}}
\end{array}\right]
\end{aligned}
$$

Corollary 6. For $p=q=1, \alpha_{1}=\sigma \in \mathbb{C}, \beta_{1}=1$, equation

(19) reduces in the following form: 


$$
\begin{aligned}
& S_{x, \infty ; r}^{\mathcal{\varepsilon}, \vartheta}\left(t^{-\eta}{ }_{1}^{\varphi, \varsigma} M_{1}\left(\sigma ; 1 ; \frac{a}{t^{\xi}}\right)\right)(x)=x^{-\eta} E_{\varphi, \varsigma}^{\sigma}\left(\frac{a}{x^{\xi}}\right) \\
& \times I_{p+2, q+1 ; r}^{m, n+2}\left[\begin{array}{c}
\lambda \mid\left(a_{j}, A_{j}\right)_{1, n} ;\left(a_{j i}, A_{j i}\right)_{n+1, p_{i}}\left(1-\frac{(\varepsilon+\eta+\xi k)}{r}, \tau\right),(-\vartheta, v) \\
\left(-\vartheta-\frac{(\varepsilon+\eta+\xi k)}{r}, \tau+v\right),\left(b_{j}, \varsigma_{j}\right)_{1, m} ;\left(b_{j i}, \varsigma_{j i}\right)_{m+1, q_{i}}
\end{array}\right] .
\end{aligned}
$$

\section{Certain Integral Transforms}

In this section, with the aid of the results developed in the prior segment, we will provide some very important outcomes of several theorems connected with the transforms of
Euler and Laplace. To this end, we would like to define these transforms first.

Definition 1. The well-known Euler transform (e.g., [19]) of a function $f(t)$ is defined as

$$
\mathfrak{B}\{f(t) ; c, d\}=\int_{0}^{1} t^{c-1}(1-t)^{d-1} f(t) \mathrm{d} t ;(c, d \in \mathbb{C}, \mathfrak{R}(c)>0, \mathfrak{R}(d)>0)
$$

Definition 2. The Laplace transform (e.g., [19]) of the function $f(t)$ is defined, as usual, by

$$
\mathfrak{L}\{f(t) ; s\}=\int_{0}^{\infty} e^{-s t} f(t) \mathrm{d} t ;(\mathfrak{R}(s)>0) .
$$

This section would establish the following fascinating outcomes in the form of theorems. As these findings are direct implications of Definitions 1 and 2 and Theorems 1 and 2, they are provided without evidence here.

Theorem 3. The Euler transform of the Theorem 1 gives the following result:

$$
\begin{aligned}
& \mathfrak{B}\left\{S_{0, x ; r}^{\mu, 9}\left(t_{p}^{\eta-1} M_{q}^{\varphi, \varsigma}\left(a t^{\xi}\right)\right) ; c, d\right\}=\sum_{k=0}^{\infty} \frac{\left(\alpha_{1}\right)_{k} \cdots\left(\alpha_{p}\right)_{k}}{\left(\beta_{1}\right)_{k} \ldots\left(\beta_{q}\right)_{k}} \frac{a^{k}}{\Gamma(\varphi k+\varsigma)} B(c+\eta-1+\xi k, d) \\
& \times I_{p+2, q+1 ; r}^{m, n+2}\left[\begin{array}{c}
\lambda \mid\left(a_{j}, A_{j}\right)_{1, n} ;\left(a_{j i}, A_{j i}\right)_{n+1, p_{i}}\left(1-\frac{(\mu+\eta+\xi k)}{r}, \tau\right),(-\vartheta, v) \\
\left(-\vartheta-\frac{(\mu+\eta+\xi k)}{r}, \tau+v\right),\left(b_{j}, \varsigma_{j}\right)_{1, m} ;\left(b_{j i}, \varsigma_{j i}\right)_{m+1, q_{i}}
\end{array}\right],
\end{aligned}
$$

provided that the conditions mentioned with the operator and Euler transform are satisfied.
Theorem 4. The Euler transform of the Theorem 2 gives the following result:

$$
\begin{aligned}
\mathfrak{B}\left\{S_{x, \infty ; r}^{\mathcal{E}, \vartheta}\left(t_{p}^{-\eta} \stackrel{M}{q}_{q}\left(\frac{a}{t^{\xi}}\right)\right) ; c, d\right\}= & \sum_{k=0}^{\infty} \frac{\left(\alpha_{1}\right)_{k} \ldots\left(\alpha_{p}\right)_{k}}{\left(\beta_{1}\right)_{k} \ldots\left(\beta_{q}\right)_{k}} \frac{a^{k}}{\Gamma(\varphi k+\varsigma)} B(c-\eta-\xi k, d) \\
& \times I_{p+2, q+1 ; r}^{m, n+2}\left[\begin{array}{l}
\left.\lambda \mid\left(a_{j}, A_{j}\right)_{1, n} ;\left(a_{j i}, A_{j i}\right)_{n+1, p_{i}},\left(1-\frac{(\varepsilon+\eta+\xi k)}{r}, \tau\right),(-\vartheta, v)\right] \\
\left.\left(-\vartheta-\frac{(\varepsilon+\eta+\xi k)}{r}, \tau+v\right),\left(b_{j}, \varsigma_{j}\right)_{1, m} ;\left(b_{j i}, \varsigma_{j i}\right)_{m+1, q_{i}}\right],
\end{array}\right.
\end{aligned}
$$


provided that the conditions mentioned with the operator and Euler transform are satisfied.
Theorem 5. The Laplace transform of the Theorem 1 gives the following result:

$$
\begin{aligned}
\mathfrak{L}\left\{S_{0, x ; r}^{\mu, \vartheta}\left(t_{p}^{\eta-1} M_{q}^{\varphi, \varsigma}\left(a t^{\xi}\right)\right) ; s\right\}= & s^{-\eta} \sum_{k=0}^{\infty} \frac{\left(\alpha_{1}\right)_{k} \cdots\left(\alpha_{p}\right)_{k}}{\left(\beta_{1}\right)_{k} \ldots\left(\beta_{q}\right)_{k}} \frac{\Gamma(\eta+\xi k)}{\Gamma(\varphi k+\varsigma)}\left(a s^{-\xi}\right)^{k} \\
& \times I_{p+2, q+1 ; r}^{m, n+2}\left[\begin{array}{l}
\left.\lambda \mid\left(a_{j}, A_{j}\right)_{1, n} ;\left(a_{j i}, A_{j i}\right)_{n+1, p_{i}},\left(1-\frac{(\mu+\eta+\xi k)}{r}, \tau\right),(-\vartheta, v)\right] \\
\left.\left(-\vartheta-\frac{(\mu+\eta+\xi k)}{r}, \tau+v\right),\left(b_{j}, \varsigma_{j}\right)_{1, m} ;\left(b_{j i}, \varsigma_{j i}\right)_{m+1, q_{i}}\right],
\end{array}\right.
\end{aligned}
$$

provided that the conditions mentioned with the operator and Laplace transform are satisfied.
Theorem 6. The Laplace transform of the Theorem 2 gives the following result:

$$
\begin{aligned}
& \mathfrak{Q}\left\{S_{x, \infty ; r}^{\mathcal{\varepsilon}, \vartheta}\left(t^{-\eta}{ }_{p}^{\varphi, \varsigma}{ }_{q}\left(\frac{a}{t^{\xi}}\right)\right) ; s\right\}=s^{\eta-1} \sum_{k=0}^{\infty} \frac{\left(\alpha_{1}\right)_{k} \ldots\left(\alpha_{p}\right)_{k}}{\left(\beta_{1}\right)_{k} \ldots\left(\beta_{q}\right)_{k}} \frac{\Gamma(1-\eta-\xi k)}{\Gamma(\varphi k+\varsigma)}\left(a s^{\xi}\right)^{k} \\
& \times I_{p+2, q+1 ; r}^{m, n+2}\left[\begin{array}{c}
\lambda \mid\left(a_{j}, A_{j}\right)_{1, n} ;\left(a_{j i}, A_{j i}\right)_{n+1, p_{i}}\left(1-\frac{(\varepsilon+\eta+\xi k)}{r}, \tau\right),(-\vartheta, v) \\
\left(-\vartheta-\frac{(\varepsilon+\eta+\xi k)}{r}, \tau+v\right),\left(b_{j}, \varsigma_{j}\right)_{1, m} ;\left(b_{j i}, \varsigma_{j i}\right)_{m+1, q_{i}}
\end{array}\right],
\end{aligned}
$$

provided that the conditions mentioned with the operator and Laplace transform are satisfied.

\section{Properties of Generalized Fractional Integral Operators}

Here, we establish some properties of the operators as consequences of Theorems 1 and 2. These properties show compositions of the power function.
Theorem 7. Following all the conditions on parameters as stated in Theorem 1 with $\mathfrak{R}(\psi+\eta)>0$, then the following result holds true:

$$
x^{\psi} S_{0, x ; r}^{\mu, \vartheta}\left(t_{p}^{\eta-1} \stackrel{\varphi, \zeta}{M}_{q}\left(a t^{\xi}\right)\right)(x)=S_{0, x ; r}^{\mu-\psi, \vartheta}\left(t^{\psi+\eta-1}{ }_{p}^{\varphi, \zeta} M_{q}\left(a t^{\xi}\right)\right)(x) .
$$

Proof. Let us use (14) in the left-hand side of (36), and we get

$$
\begin{aligned}
x^{\psi} S_{0, x ; r}^{\mu, 9}\left(t_{p}^{\eta-1} M_{q}^{\varphi, \zeta}\left(a t^{\xi}\right)\right)(x)= & \sum_{k=0}^{\infty} \frac{\left(\alpha_{1}\right)_{k} \ldots\left(\alpha_{p}\right)_{k}}{\left(\beta_{1}\right)_{k} \ldots\left(\beta_{q}\right)_{k}} \frac{(a)^{k}}{\Gamma(\varphi n+\varsigma)} x^{\eta+\psi+\xi k-1} \\
& \times I_{p+2, q+1 ; r}^{m, n+2}\left[\begin{array}{l}
\left.\lambda \mid\left(a_{j}, A_{j}\right)_{1, n} ;\left(a_{j i}, A_{j i}\right)_{n+1, p_{i}},\left(1-\frac{(\mu+\eta+\xi k)}{r}, \tau\right),(-\vartheta, v)\right] \\
\left.\left(-\vartheta-\frac{(\mu+\eta+\xi k)}{r}, \tau+v\right),\left(b_{j}, \varsigma_{j}\right)_{1, m} ;\left(b_{j i}, \varsigma_{j i}\right)_{m+1, q_{i}}\right]
\end{array} .\right.
\end{aligned}
$$

Again, using (14) in the right-hand side of (37), we get 


$$
\begin{aligned}
& S_{0, x ; r}^{\mu-\psi, \vartheta}\left(t^{\psi+\eta-1}{ }_{p}^{\varphi, \varsigma}{ }_{q}^{M}\left(a t^{\xi}\right)\right)(x)=\sum_{k=0}^{\infty} \frac{\left(\alpha_{1}\right)_{k} \cdots\left(\alpha_{p}\right)_{k}}{\left(\beta_{1}\right)_{k} \ldots\left(\beta_{q}\right)_{k}} \frac{(a)^{k}}{\Gamma(\varphi k+\varsigma)} x^{\eta+\psi+\xi k-1} \\
& \times I_{p+2, q+1 ; r}^{m, n+2}\left[\begin{array}{c}
\lambda \mid\left(a_{j}, A_{j}\right)_{1, n} ;\left(a_{j i}, A_{j i}\right)_{n+1, p_{i}},\left(1-\frac{(\mu+\eta+\xi k)}{r}, \tau\right),(-\vartheta, v) \\
\left(-\vartheta-\frac{(\mu+\eta+\xi k)}{r}, \tau+v\right),\left(b_{j}, \varsigma_{j}\right)_{1, m} ;\left(b_{j i}, \varsigma_{j i}\right)_{m+1, q_{i}}
\end{array}\right] . \\
& x^{-\psi} S_{x, \infty ; \gamma, r}^{\varepsilon, 9}\left(t_{p}^{-\eta}{ }_{q}^{\varphi, \zeta}\left(\frac{a}{t^{\xi}}\right)\right)(x)=S_{x, \infty ; r, r}^{\varepsilon-\psi, \vartheta}\left(t^{-\psi-\eta}{ }_{p}^{\varphi, \zeta}{ }_{q}\left(\frac{a}{t^{\xi}}\right)\right)(x) .
\end{aligned}
$$
(38).

Theorem 8. Follow all the conditions on parameters as stated in Theorem 2 with $\mathfrak{R}(1-\psi+\eta)<1$; then, the following result holds true:

Proof. From (14) in the left-hand side of (39), we get

$$
\begin{aligned}
& x^{-\psi} S_{x, \infty ; r}^{\varepsilon, \vartheta}\left(t^{-\eta} \stackrel{\varphi, \zeta}{M}_{q}\left(\frac{a}{t^{\xi}}\right)\right)(x)=\sum_{k=0}^{\infty} \frac{\left(\alpha_{1}\right)_{k} \cdots\left(\alpha_{p}\right)_{k}}{\left(\beta_{1}\right)_{k} \ldots\left(\beta_{q}\right)_{k}} \frac{(a)^{k}}{\Gamma(\varphi k+\varsigma)} x^{-\eta-\psi-\xi k} \\
& \times I_{p+2, q+1 ; r}^{m, n+2}\left[\begin{array}{c}
\lambda \mid\left(a_{j}, A_{j}\right)_{1, n} ;\left(a_{j i}, A_{j i}\right)_{n+1, p_{i}},\left(1-\frac{(\varepsilon+\eta+\xi k)}{r}, \tau\right),(-\vartheta, v) \\
\left(-\vartheta-\frac{(\varepsilon+\eta+\xi k)}{r}, \tau+v\right),\left(b_{j}, \varsigma_{j}\right)_{1, m} ;\left(b_{j i}, \varsigma_{j i}\right)_{m+1, q_{i}}
\end{array}\right] .
\end{aligned}
$$

Again, using (14) in the right-hand side of (39), we get

$$
\begin{aligned}
& S_{x, \infty ; r}^{\mathcal{E}-\psi, \vartheta}\left(t^{-\psi-\eta}{ }_{p}^{\varphi, \varsigma}{ }_{q}\left(\frac{a}{t^{\xi}}\right)\right)(x)=\sum_{k=0}^{\infty} \frac{\left(\alpha_{1}\right)_{k} \cdots\left(\alpha_{p}\right)_{k}}{\left(\beta_{1}\right)_{k} \ldots\left(\beta_{q}\right)_{k}} \frac{(a)^{k}}{\Gamma(\varphi k+\varsigma)} x^{-\eta-\psi-\xi k} \\
& \times I_{p+2, q+1 ; r}^{m, n+2}\left[\begin{array}{c}
\lambda \mid\left(a_{j}, A_{j}\right)_{1, n} ;\left(a_{j i}, A_{j i}\right)_{n+1, p_{i}},\left(1-\frac{(\varepsilon+\eta+\xi k)}{r}, \tau\right),(-\vartheta, v) \\
\left(-\vartheta-\frac{(\varepsilon+\eta+\xi k)}{r}, \tau+v\right),\left(b_{j}, \varsigma_{j}\right)_{1, m} ;\left(b_{j i}, \varsigma_{j i}\right)_{m+1, q_{i}}
\end{array}\right] .
\end{aligned}
$$
(41).

It seems that Theorem 7 readily follows due to (40) and

\section{Concluding Remark and Observations}

In this study, we introduced and studied the properties of generalized $M$-series under the new (presumed) generalized fractional integral operators which are defined in equations (6) and (7) and also developed some new images. The results established in this study contain various special cases, such that if we take $r=1$, we recover the known results recorded in [20]. Furthermore, we can present certain very interesting results in the form of several theorems associated with Mellin, Whittaker, and $K$-transforms. We left this as an exercise to the interested reader.

\section{Data Availability}

No data were used to support this study.

\section{Conflicts of Interest}

The authors declare that there are no conflicts of interest. 


\section{References}

[1] M. Sharma and R. Jain, "A note on a generalized $M$-series as a special function of fractional calculus," Fractional Calculus and Applied Analysis, vol. 12, no. 4, pp. 449-452, 2009.

[2] A. A. Kilbas, H. M. Srivastava, and J. J. Trujillo, Theory and Applications of Fractional Differential Equations, Elsevier, North-Holland Mathematics Studies, Amsterdam, Netherlands, 2006.

[3] C. Fox, "The $G$-and $H$-functions as symmetrical Fourier kernels," Transactions of the American Mathematical Society, vol. 98, no. 3, p. 395, 1961.

[4] A. M. Mathai and R. K. Saxena, The H-Function with Applications in Statistics and Other Disciplines, Wiley Eastern Ltd., New Delhi, India, 1978.

[5] H. M. Srivastava, K. C. Gupta, and S. P. Goyal, The HFunctions on One and Two Variables, South Asian Publisher's, New Delhi, India, 1981.

[6] M. Kumar Bansal, D. Kumar, I. Khan, J. Singh, and K. S. Nisar, "Certain unified integrals associated with product of $M$-series and incomplete $H$-functions," Mathematics, vol. 7, no. 12, p. 1191, 2019.

[7] M. Kumar Bansal, D. Kumar, K. S. Nisar, and J. Singh, "Certain fractional calculus and integral transform results of incomplete $\aleph$-functions with applications," Mathematical Methods in the Applied Sciences, vol. 43, no. 4, pp. 5602-5614, 2020.

[8] Y. Singh, V. Gill, J. Singh, D. Kumar, and I. Khan, "Computable generalization of fractional kinetic equation with special functions," Journal of King Saud University-Science, vol. 33, no. 1, p. 101221, 2021.

[9] J. Singh, D. Kumar, and D. Baleanu, "New aspects of fractional Bloch model associated with composite fractional derivative," Mathematical Modelling of Natural Phenomena, vol. 16, p. 10, 2021.

[10] D. L. Suthar, R. K. Parmar, and S. D. Purohit, "Fractional calculus with complex order and generalized hypergeometric functions," Nonlinear Science Letters A, vol. 8, no. 2, pp. 156-161, 2017.

[11] G. E. Andrews, R. Askey, and R. Roy, Special Functions, Cambridge University Press, Cambridge, UK, 1999.

[12] H. Amsalu and D. L. Suthar, "Generalized fractional integral operators involving Mittag-Leffler function," Abstract and Applied Analysis, vol. 2018, Article ID 7034124, 8 pages, 2018.

[13] G. M. Mittag-Leffler, "Sur la nouvelle function $E_{\alpha}(x)$," Comptes Rendus de l'Académie des Sciences Paris (Series II), vol. 137, pp. 554-558, 1903.

[14] A. Wiman, "Über den fundamental satz in der theorie der functionen $E_{\alpha}(x)$," Acta Mathematica, vol. 29, pp. 191-201, 1905.

[15] T. R. Prabhakar, "A singular integral equation with a generalized Mittag-Leffler function in the kernel," Yokohama Mathematical Journal, vol. 19, pp. 7-15, 1971.

[16] R. K. Saxena and R. K. Kumbhat, "Integral operators involving $H$-function," Indian Journal of Pure and Applied Mathematics, vol. 5, pp. 1-6, 1974.

[17] D. L. Suthar, "Generalized fractional calculus operators associated with K-function," International Journal of Mathematical Physics, vol. 1, no. 2, pp. 1-11, 2018.

[18] V. P. Saxena, The I-Function, Anamaya Publisher, New Delhi, India, 2008.

[19] I. N. Sneddon, The Use of Integral Transform, Tata Mc Graw Hill, New Delhi, India, 1979.
[20] A. M. Khan, R. K. Kumbhat, A. Chouhan, and A. Alaria, "Generalized fractional integral operators and M-Series," Journal of Mathematics, vol. 2016, Article ID 2872185, 10 pages, 2016. 